\title{
Prevalence of Sarcopenic Obesity in Adults with Class II/III Obesity Using Different Diagnostic Criteria
}

\author{
Carlene A. Johnson Stoklossa, ${ }^{1}$ Arya M. Sharma, ${ }^{2}$ Mary Forhan, ${ }^{3}$ \\ Mario Siervo, ${ }^{4}$ Raj S. Padwal, ${ }^{5}$ and Carla M. Prado ${ }^{1}$ \\ ${ }^{1}$ Department of Agriculture, Food and Nutritional Science, University of Alberta, Edmonton, AB, Canada \\ ${ }^{2}$ Department of Medicine, University of Alberta, Edmonton, AB, Canada \\ ${ }^{3}$ Department of Occupational Therapy, University of Alberta, Edmonton, AB, Canada \\ ${ }^{4}$ Human Nutrition Research Centre, Institute of Cellular Medicine, Newcastle University, Newcastle on Tyne, UK \\ ${ }^{5}$ Department of Medicine, University of Alberta, Alberta Diabetes Institute, Edmonton, AB, Canada
}

Correspondence should be addressed to Carla M. Prado; cprado@ualberta.ca

Received 26 July 2016; Accepted 12 February 2017; Published 22 March 2017

Academic Editor: Pedro Moreira

Copyright (C) 2017 Carlene A. Johnson Stoklossa et al. This is an open access article distributed under the Creative Commons Attribution License, which permits unrestricted use, distribution, and reproduction in any medium, provided the original work is properly cited.

Background/Objective. Sarcopenic obesity (SO) is a hidden condition of reduced lean soft tissue (LST) in context of excess adiposity. SO is most commonly reported in older adults and both its risk and prevalence increase with age. A variety of body composition indices and cut points have been used to define this condition, leading to conflicting prevalence and risk prediction. Here, we investigate variability in the prevalence of SO in an adult sample of individuals with class II/III obesity $\left(\mathrm{BMI} \geq 35 \mathrm{~kg} / \mathrm{m}^{2}\right) \mathrm{using}$ different diagnostic criteria. Methods. SO definitions were identified from a literature review of studies using dual-energy X-ray absorptiometry (DXA) to assess LST. Demographics, anthropometrics, and body composition (by DXA) were measured in $n=120$, $86 \%$ female ( $46.9 \pm 11.1$ years). Results. LST was extremely variable in individuals, even with similar body sizes, and observed across the age spectrum. The prevalence of SO ranged from 0 to $84.5 \%$ in females and 0 to $100 \%$ in males, depending upon the definition applied, with higher prevalence among definitions accounting for measures of body size or fat mass. Conclusion. SO is present, yet variable, in adults with class II/III obesity. Accounting for body mass or fat mass may identify a higher number of individuals with $\mathrm{SO}$, although risk prediction remains to be studied.

\section{Introduction}

Obesity is a complex chronic global disease affecting people worldwide across all ages, sexes, ethnicities, and nationalities [1]. Comparing body weight to height using body mass index (BMI) allows for the classification of obesity into class I (BMI $30-34.9 \mathrm{~kg} / \mathrm{m}^{2}$ ), class II (BMI $35-39.9 \mathrm{~kg} / \mathrm{m}^{2}$ ), and class III $\left(B M I \geq 40 \mathrm{~kg} / \mathrm{m}^{2}\right)$ [2] The prevalence of the highest obesity class is of concern due to its association with poorer health outcomes compared to lower BMI categories [1]. In 20132014, class III obesity affected $5.5 \%$ of males and $9.9 \%$ of females in the United States, with a linear increase in prevalence for females since 2005 [3]. Compared to the United States, fewer Canadians have class III obesity (2.5\%); however the same trend by sex is found with a greater percentage of females affected (3\%, compared to $2 \%$ of men) $[4,5]$.

In addition to BMI, waist circumference can be used to identify obesity. Both anthropometric methods provide a surrogate assessment of fat mass (FM) but are poor detectors of lean mass, also called lean soft tissue (LST), and hence of body composition. People with obesity can have varying proportions of LST and the single use of anthropometry to diagnose obesity precludes an accurate characterization of the different proportions of FM versus LST of an individual [6].

Emerging evidence suggests obesity can coexist with low LST (sarcopenia) [7-10]. In this case, the gravity impact of the excess body weight may not be sufficient to promote a concurrent increase in LST; therefore, individuals with obesity 
may have high FM without a parallel increase in LST [11]. Notably, this phenotype termed sarcopenic obesity may not be identified without the use of body composition assessment techniques. Low LST is an important prognostic factor in health and clinical conditions, as its main component is skeletal muscle mass, a tissue of vital importance for strength, functional mobility, immune function, and wound healing, among others $[8,12-16]$.

Sarcopenia has been primarily studied in older adults and individuals with chronic conditions but emerging evidence suggests that "healthy," younger individuals are also at risk for presenting with this condition $[17,18]$. Compounded with the consequences of excess FM, the concurrent sarcopenic obesity phenotype has been independently associated with worse morbidity and disability than either sarcopenia or obesity alone [10]. In the context of obesity treatment, weight loss results in the loss of both FM and LST [19, 20]. With repeated weight loss-gain cycles combined with age-related body composition changes, developing sarcopenic obesity is possible [10].

The identification of sarcopenic obesity is limited not only due to the availability of accurate body composition techniques but also due to heterogeneity in its diagnostic criteria [10]. A variety of body composition indices and cut-offs have been used to define sarcopenia and obesity, leading to conflicting findings on the prevalence and risk prediction of this condition $[10,21]$ Additionally, the great majority of studies have focused on identifying sarcopenic obesity in older adults and the prevalence within younger adults and those with class II/III obesity is not well defined. With the increasing prevalence of class III obesity [3] and of sarcopenia [18], the prevalence of sarcopenic obesity in these individuals is likely to increase dramatically. Therefore, the objective of this study is to explore the variability in the prevalence of sarcopenic obesity in an adult sample with class II/III obesity using different diagnostic criteria.

\section{Methods}

In a cross-sectional approach, we included consecutive patients from a multidisciplinary clinic providing medical and bariatric surgical interventions for adults (18-69 years) with class II/III obesity (BMI $\geq 35 \mathrm{~kg} / \mathrm{m}^{2}$ ) in Edmonton, Alberta, Canada. Ethics approval was received from the University of Alberta Health Research Ethics Board.

Medical records were used to assess demographic and medical history obtained from the initial clinic assessment. Height was measured (without shoes, within $0.1 \mathrm{~cm}$ ) with a wall-mounted stadiometer. Weight was measured (single layer of clothing, without shoes, within $0.1 \mathrm{~kg}$ ) with a highcapacity weigh scale (Scale-Tronix $6702 \mathrm{~W}^{\circledR}$, WelchAllyn Inc., Skaneateles Falls, New York). Waist circumference was measured (within $0.1 \mathrm{~cm}$ ) with a nonstretch tape at the midpoint of the torso (between lowest rib and iliac crest) on the right side using a cross-handed technique, recorded as the average of three consecutive measures.

Dual-energy X-ray absorptiometry (DXA) was required at the initial assessment and completed at a local medical imaging center [Hologic Discovery A (S/N 45026) or W (S/
N 83792) scanners, software version 12.7.4.2, Hologic Inc., Bedford MA]. No subjects exceeded the DXA weight capacity limit $(204 \mathrm{~kg})$ or scan area length $(195 \mathrm{~cm})$. Reflection positioning was used for subjects with larger supine widths ( $>65 \mathrm{~cm}$ ). Right side data was duplicated when values for the left side were either not reliable or available [22-24]. Collected values included whole body and segmental values for FM, LST, appendicular skeletal muscle mass (ASM, which is LST from arms and legs), and fat-free mass (FFM $=$ LST + bone), and its derivatives are adjusted by height in square meters, also called indexes (e.g., FMI, ASMI). Detailed definitions of each of these body composition variables can be found elsewhere [6].

Subjects with complete initial clinic assessments and body composition analysis by DXA were included in the study. DXA scans available for analysis dated from January 2009 to June 2012, after which they were no longer ordered at the initial clinical assessment. All data was collected prior to starting obesity treatment. Subjects were excluded from the final analysis if DXA data was unreliable (i.e., segmental measurements were outside of the field of view or due to lack of separation of tissues between the arms and torso).

2.1. Sarcopenic Obesity: Definitions and Terminology. A literature search was conducted using PubMed, Scopus, and Web of Science databases to identify studies using definitions sarcopenic obesity based upon body composition data derived from DXA with or without use of anthropometric variables (e.g., weight, BMI, and waist circumference), excluding clinical studies (e.g., cancer). For definitions using ethnic-specific cut points, white/Caucasian references were included as the majority of our population (83.9\% Edmonton, 86\% Canada) self-identified as Caucasian [25]. Ethnicity was not collected as part of the clinic assessment, in accordance with the Freedom of Information and Protection of Privacy Act [26], therefore unavailable for analysis.

Based on the literature review, ten studies were identified using nine variables based upon LST or ASM to define sarcopenia (Table 1) and four variables were identified to define obesity (Table 2, plus FMI phenotype listed in Table 1) [7, 8, $18,27-33]$.

The use of inconsistent body composition terminology may preclude a clear understanding of sarcopenic obesity's diagnostic criteria in the literature (i.e., authors use of different terminology for the same body composition variables). Therefore, in order to improve clarity while still accurately representing the body composition components being measured in each study, we consistently use the terms LST for studies measuring the nonbone, nonfat body compartment in general from the whole body (i.e., arms, legs, trunk, and head) and ASM for studies measuring LST from the arms and legs [6].

With the exception of BMI, each variable for sarcopenia and obesity used sex-specific cut points, with more than one cut point for some variables. Sixteen unique definitions (composed of a variable and cut point for each sarcopenia and obesity) were identified and applied to the sample to 


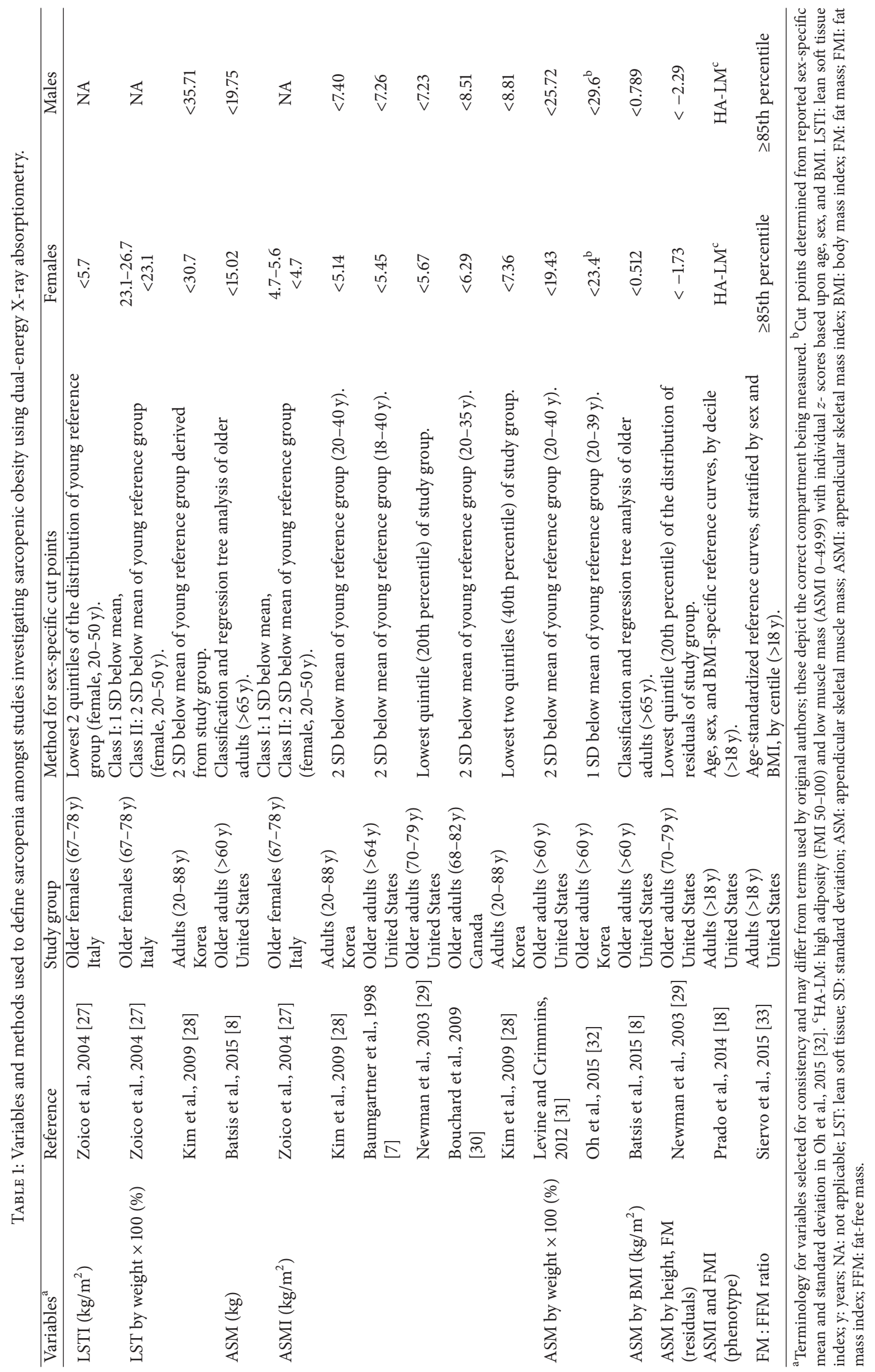


TABLE 2: Prevalence of obesity in study cohort $(n=120)$ using various sex-specific definitions determined by anthropometric and dual-energy $\mathrm{X}$-ray absorptiometry measurements amongst studies investigating sarcopenic obesity.

\begin{tabular}{llccc}
\hline Variables & Reference & \multicolumn{2}{c}{ Females $(n=103)$} & \multicolumn{2}{c}{ Males $(n=17)$} \\
Prevalence (\%)
\end{tabular}

${ }^{\mathrm{a}}$ Waist circumference not available for the entire cohort: females $(n=81,78.6 \%)$ and males $(n=13,76.5 \%)$. BMI: body mass index; NA: not applicable.

explore the prevalence of sarcopenic obesity. Linear regression analysis with ASM, height, and FM (kg) was used to determine prevalence of sarcopenia using the Newman et al., residual method [29]. The classification by body composition phenotypes was determined using deciles of populationderived ASMI and FMI cut points based on sex, BMI, and age, as per the protocol described in Prado et al. [18]. The classification of abnormal body composition phenotype as a load-capacity model (load being FM and capacity FFM) was calculated as the ratio of FM:FFM (as centiles), as per methodology described in Siervo et al. [33].

Additional classifications were derived from our study cohort, using ASMI calculated as the lowest 20th percentile and two standard deviations (SD) below the mean of the distribution; a method commonly reported in the literature when a reference population is not available [34]. Definitions of sarcopenic obesity utilizing measures of muscle strength or function were not included, as data were not available for our cohort.

2.2. Statistical Analysis. Descriptive statistics were used for subject characteristics, anthropometrics, and body composition and reported as mean (interquartile range). Normality testing was completed using the Shapiro-Wilk test. Frequencies and proportions were reported for categorical variables. Independent samples $t$-test for normally distributed data and nonparametric (Mann-Whitney $U$ ) independent samples $t$-test were used to compare variables between sexes. To account for missing data (waist circumference), subjects were compared to determine if differences existed between the groups. Correlations were tested using Pearson's $r$ to explore the relationship between variables. Two-tailed tests were used for all the analysis with a $p$ value of $<0.05$ considered for statistical significance. Data was analysed using IBM SPSS Statistics for Mac, version 23 (IBM Corp., Armonk, NY, USA).

\section{Results}

A total of 167 subjects with completed initial assessments and DXA scans were initially reviewed, in which 120 subjects
(85.8\% female) had reliable DXA data to be included in the final analysis. Clinical characteristics of excluded subjects $(n=47)$ were not different from those included in the analysis. Mean age of the entire cohort was $46.9 \pm 11.1$ years (range: 18-69 years). Subject characteristics, anthropometrics, and body composition are presented in Table 3.

Patients were community-dwelling (100\%) and predominantly married/common-law (F 68\%, M 65\%) and worked outside the home (F 68\%, M 70\%) and 7.8\% of females (no males) were current smokers. The majority of patients were generally well educated (F 98\%, M 94\% completed high school), with more females than males who completed their education at a university/college level (F 57\%, M 35\%). Recommended activity levels ( $>150$ minutes of moderate intensity activities a week) were met by $20 \%$ of females and $23 \%$ of males.

Due to the positively skewed data for weight in females, some variables were not normally distributed. Independent samples $t$-tests and nonparametric (Mann-Whitney $U$ ) tests results were compared and showed the same results. No significant differences were observed between females and males for age, BMI, and FM (kg), Table 3. Compared to males, females had higher values for FM (\%), FMI, and FM : FFM ratio and lower values for variables depicting the lean mass compartment. A large variability in LST (kg) was observed for individuals with the same body size, Figures 1(a) and 1(b). The relationship between BMI and LST in females and males was moderate and weak ( $R=0.41, R=0.20$, resp.), Figure 1 (a).

The entire cohort met the criteria for obesity defined by BMI, waist circumference, and FMI cut points (Table 2). For FM\%, all males exceeded the five different cut points. One female (BMI $39.7 \mathrm{~kg} / \mathrm{m}^{2}$ and $32.2 \% \mathrm{FM}$ ) did not meet the criteria for obesity defined by \% FM with five of the six different cut points. Ten females (9.7\%) had \% FM below the highest cut point (42.9\%) and therefore would not be identified with obesity despite BMI's ranging from 35.9 to $45.1 \mathrm{~kg} / \mathrm{m}^{2}$. Of note, the highest sex-specific 20 th percentile for FMI was $>23.8 \mathrm{~kg} / \mathrm{m}^{2}$ for females and $>21.5 \mathrm{~kg} / \mathrm{m}^{2}$ for males.

Considering the entire cohort had class II/III obesity as defined by BMI, when each definition of sarcopenia was 
TABLE 3: Subject characteristics, anthropometrics, and body composition $(n=120)$, by sex.

\begin{tabular}{|c|c|c|c|}
\hline Variables $^{\mathrm{a}}$ & $\begin{array}{c}\text { Females }(n=103) \\
\text { Mean }(\mathrm{IQR})\end{array}$ & $\begin{array}{c}\text { Males }(n=17) \\
\text { Mean }(\mathrm{IQR})\end{array}$ & $p$ value \\
\hline Age (years) & $46.5(18)$ & $49.4(10)$ & 0.352 \\
\hline \multicolumn{4}{|c|}{ Anthropometrics } \\
\hline Height $(\mathrm{cm})$ & $164.1(8.3)$ & $177.2(9.7)$ & $<0.0001$ \\
\hline Weight (kg) & $117.3(25.8)^{b}$ & $138.2(24.9)$ & $<0.0001$ \\
\hline $\operatorname{BMI}\left(\mathrm{kg} / \mathrm{m}^{2}\right)$ & $43.5(8.4)^{\mathrm{b}}$ & $44.0(6.3)$ & 0.960 \\
\hline Waist $(\mathrm{cm})^{\mathrm{c}}$ & $120.4(18.2)$ & $141.0(15.3)$ & $<0.0001$ \\
\hline \multicolumn{4}{|c|}{ Body composition } \\
\hline Fat mass (kg) & $55.6(16.8)^{\mathrm{b}}$ & $56.5(17.5)$ & 0.759 \\
\hline Fat mass $(\%)$ & $48.0(5.7)$ & $41.4(8.3)$ & $<0.0001$ \\
\hline FMI $\left(\mathrm{kg} / \mathrm{m}^{2}\right)$ & $20.6(5.1)^{\mathrm{b}}$ & $18.0(6.0)$ & 0.009 \\
\hline FM : FFM ratio & $0.9(0.2)$ & $0.7(0.2)$ & $<0.0001$ \\
\hline LST $(\mathrm{kg})$ & $57.1(9.3)^{\mathrm{b}}$ & $76.2(13.9)$ & $<0.0001$ \\
\hline LSTI $\left(\mathrm{kg} / \mathrm{m}^{2}\right)$ & $21.2(2.6)^{\mathrm{b}}$ & $24.2(3.9)$ & $<0.0001$ \\
\hline LST by weight $\times 100(\%)$ & $49.0(6.4)$ & $55.4(9.1)$ & $<0.0001$ \\
\hline ASM $(k g)$ & $24.7(4.8)^{\mathrm{b}}$ & $34.2(7.3)$ & $<0.0001$ \\
\hline $\operatorname{ASMI}\left(\mathrm{kg} / \mathrm{m}^{2}\right)$ & $9.2(1.6)$ & $10.9(2.3)$ & $<0.0001$ \\
\hline ASM by weight $\times 100(\%)$ & $21.2(2.9)$ & $24.9(5.3)$ & $<0.0001$ \\
\hline ASM by BMI & $0.57(0.1)^{\mathrm{b}}$ & $0.78(0.2)$ & $<0.0001$ \\
\hline
\end{tabular}

${ }^{\mathrm{a}}$ Terminology for variables is selected for consistency and may differ from terms used by original authors. ${ }^{\mathrm{b}}$ Variable not normally distributed. ${ }^{\mathrm{c}}$ Waist circumference not available for the entire cohort: females $(n=81,78.6 \%)$ and males $(n=13,76.5 \%)$. IQR: interquartile range; BMI: body mass index; FMI: fat mass index; FM: fat mass; FFM: fat-free mass; LST: lean soft tissue; LSTI: lean soft tissue index; ASM: appendicular skeletal mass; ASMI: appendicular skeletal mass index.

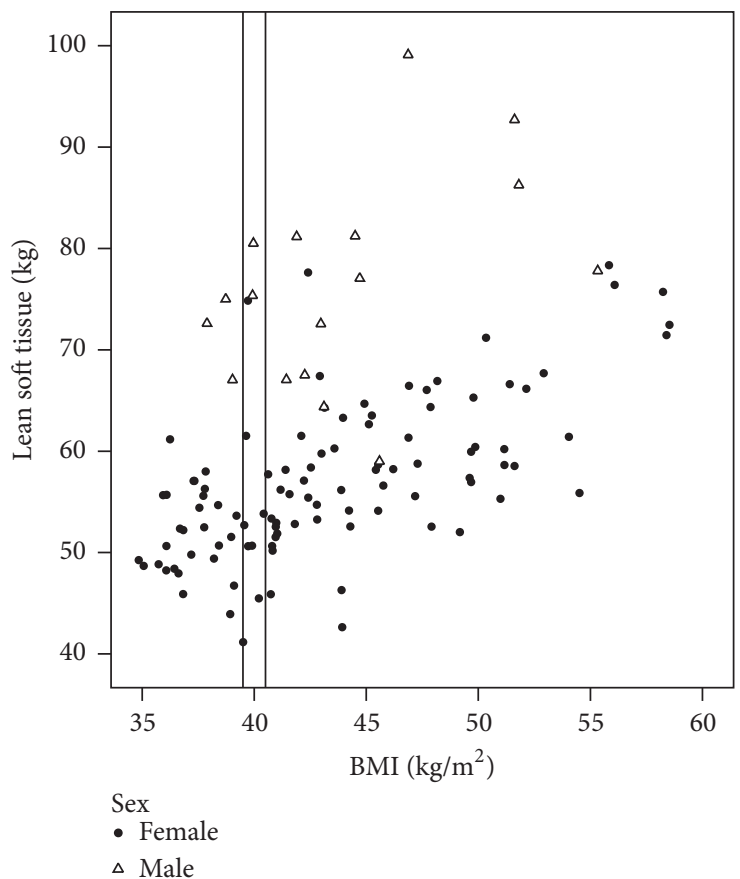

(a)

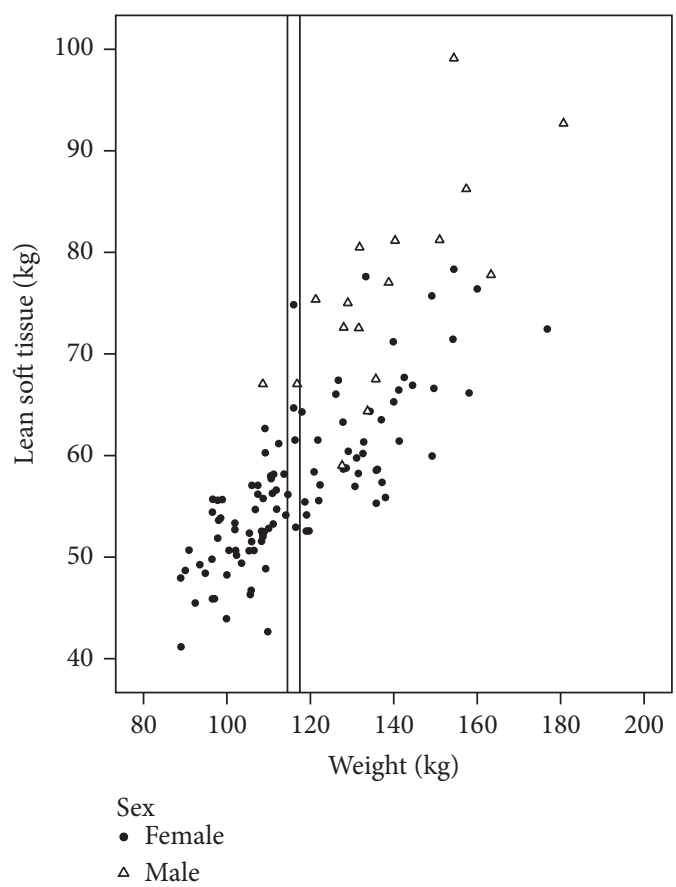

(b)

FIGURE 1: Variability of lean soft tissue by (a) body mass index (BMI) and (b) weight in adults with class II/III obesity $(n=120$, females $=$ 103). The box illustrates selected examples of females with (a) the same BMI (40 kg/m²) but LST varying from 41.2 to $74.9 \mathrm{~kg}$ and (b) same weight $(116 \mathrm{~kg}$ ) but LST varying from 52.9 to $74.9 \mathrm{~kg}$. 
applied to the current sample, the prevalence of sarcopenic obesity varied from 0 to $84.5 \%$ for females and 0 to $100 \%$ for males (Table 4). Definitions using unadjusted values for LST, ASM, or ASMI, with the exception of the highest ASMI cut point, failed to identify any subjects with sarcopenic obesity. Notably, a higher prevalence of sarcopenic obesity was identified by definitions combining ASM either with weight, BMI, or a measure of FM.

The sex-specific cut points developed from the Newman et al. [29] study group were only able to identify males with sarcopenic obesity in our cohort, Table 4. Applying the Newman et al. [29] residual method to derive cut points from the current cohort, sarcopenic obesity was identified in both sexes. For the latter, the cohort-specific cut points derived from the 20th percentile of the sex-specific distributions of the residuals were $<2.96$ for females and $<-4.82$ for males, identifying $19.4 \%$ of females and $17.6 \%$ of males with sarcopenic obesity.

Equivalent cut points for ASMI were also derived from the study cohort. The cohort-specific 20th percentile cut point to describe sarcopenic obesity by ASMI was $<8.21 \mathrm{~kg} /$ $\mathrm{m}^{2}$ for females and $<9.44 \mathrm{~kg} / \mathrm{m}^{2}$ for males. Using the lowest 2 SD criteria for ASMI, the cohort-specific cut points were $<6.79 \mathrm{~kg} / \mathrm{m}^{2}$ for females and $<8.62 \mathrm{~kg} / \mathrm{m}^{2}$ for males. Selecting the cohort-specific 20th percentile, low ASMI was observed across the age spectrum, Figure 2.

Using the phenotype definition proposed by Prado et al. [18] to the entire sample, $16(13.3 \%)$ subjects where classified with high adiposity and low muscularity (sarcopenic obeselike phenotype), and 95 (79.2\%) subjects presented with the high adiposity and high muscularity phenotype (obese nonsarcopenic-like phenotype), Figure 3. Nine females were classified as having a normal body composition phenotype.

Using the load-capacity model to account for the interaction of both body compartments [33], the FM : FFM ratio identified about a third of females and three-quarters of males with moderate and severe body composition phenotype ( $\geq 85$ th percentile), Table 4 .

\section{Discussion}

Previous research identified sarcopenic obesity in older adults [35] and groups with certain chronic diseases [6]. Although several diagnostic criteria have been used, no one approach has been widely accepted. This is the first study to use stateof-the-art methodology (DXA) to explore the prevalence of sarcopenia in a younger adult cohort (mean age $46.9 \pm$ 11.1 years) with class II/III obesity. LST was extremely variable in individuals with similar BMI illustrating a wide variability of body composition within the same body size. Using 16 previously reported definitions, the prevalence of sarcopenic obesity varied from zero to $100 \%$. Such variability precludes a comprehensive understanding of the prevalence of sarcopenia in younger individuals with more severe classes of obesity, as well as the development of preventive and treatment strategies for this condition in clinical settings. As these individuals are actively seeking obesity treatment, maintaining lean mass should be a coprimary endpoint of the nutrition care plan together with weight management.
All males and almost all females were classified with obesity using diverse FM\% cut points (30-42.9\% for females and $20-29 \%$ for males). Most individuals with a BMI $\geq$ $35 \mathrm{~kg} / \mathrm{m}^{2}$, excluding extremely muscular individuals, will present with excess adiposity [36] and prevalence will vary only based on the comparison cohort used to identify the cut point. For example, 10 females from our cohort would not be considered to have obesity using the Zoico et al. [27] cut point based on quintiles of \% FM from a sample of healthy elderly females (BMI $26 \pm 3.8 \mathrm{~kg} / \mathrm{m}^{2}$ ). Nonetheless, these 10 females were within $0.2 \%$ to $3.7 \%$ below the $\%$ FM cut point. Interestingly, using the adjusted Prado et al. cut points [18], we observed that nine females were not classified as having high adiposity. In addition to sex, this cut point is notably adjusted for age and BMI. Six females were identified as having both lower \% FM and FMI using the Zoico et al. [27] and Prado et al. [18] cut points respectively.

Prevalence of sarcopenia ranged approximately from 0 to $84.5 \%$ in females and from 0 to $100 \%$ in males. The null prevalence using several cut points may be explained by the approach used to define sarcopenia. Reference values to diagnose sarcopenia have been primarily developed from older cohorts, which may not be applicable for younger adults. Although Cherin et al. [17] included younger individuals (45-83 years), their cohort's mean age was $63.1 \pm$ 10.2 years and the prevalence of sarcopenic obesity was not reported.

In our study, no subjects were identified with sarcopenic obesity by definitions of LST [27, 28]. Baumgartner et al. $[7,37]$ and others have defined sarcopenia using ASMI sexspecific cut points based on two standard deviations below the mean for a young reference group [7, 21, 30, 37-39]. No subjects were identified with sarcopenic obesity applying each of these different cut points. Although these young reference groups were North American and of similar age to the current study cohort, their BMI (described as "normal") would be much lower. However, sarcopenic obesity may still be present but not identified as the cut points may not be sensitive enough to identify relatively low lean mass in subjects with larger total body mass. Likewise, no subjects were identified as sarcopenic using Newman et al. cut point which defined sarcopenia as the lowest 20th percentile of their cohort's ASMI distribution [29]. Notably, applying the same method to our cohort, our ASMI cut points were $45 \%$ and $31 \%$ greater for females and males, respectively, highlighting how differences in age and body size may impact comparison among different cohorts.

Although the quantity of LST may meet or exceed reference values derived from normal, healthy reference populations (e.g., normal BMI or age 25 years), the higher LST amount is insufficient to maintain the larger body size (largely due to a larger FM amount). This phenomenon can be conceptualized as the metabolic load (due to FM) versus capacity (of the LST/FFM) model previously described [33]. Therefore, sarcopenia in those with obesity may be present at higher LST values and must be evaluated in relation to body mass or FM. 


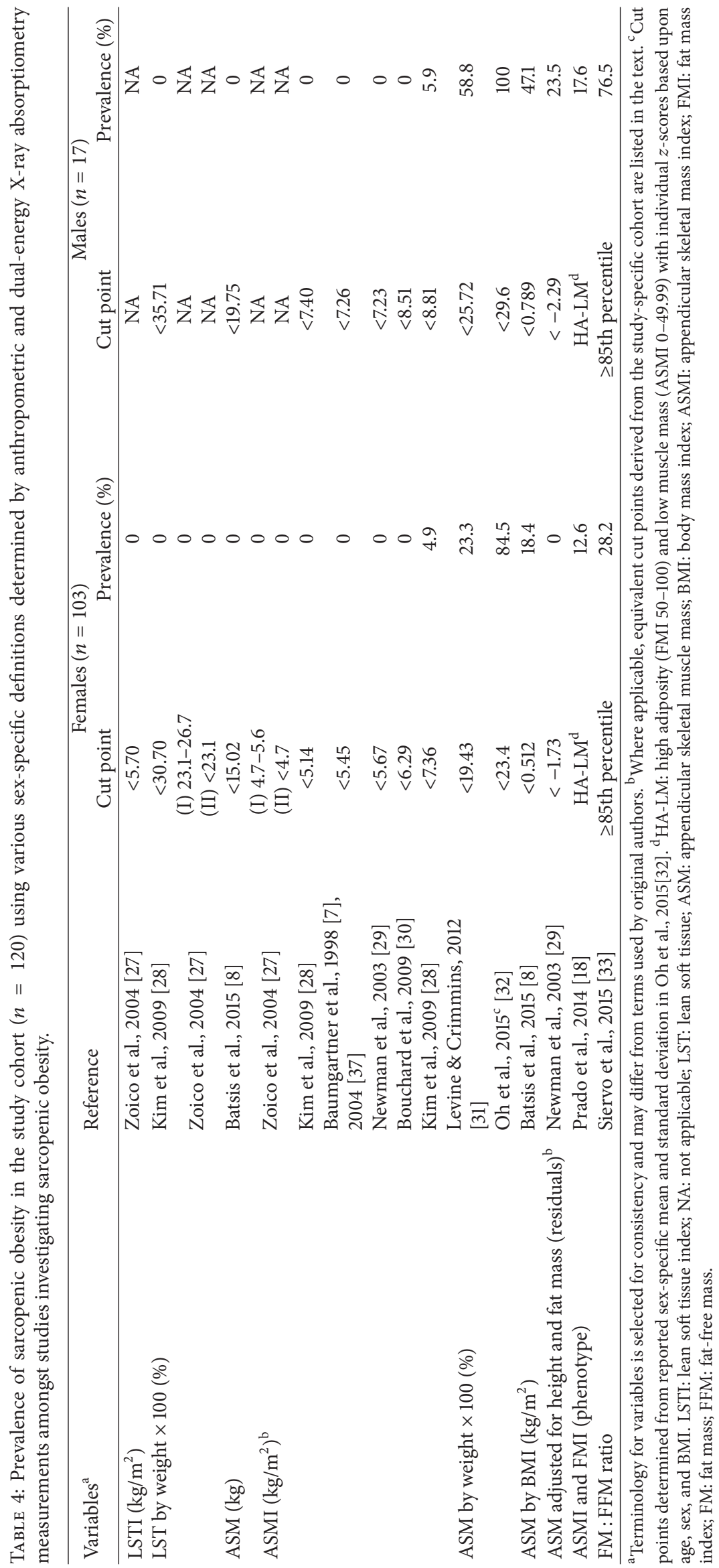




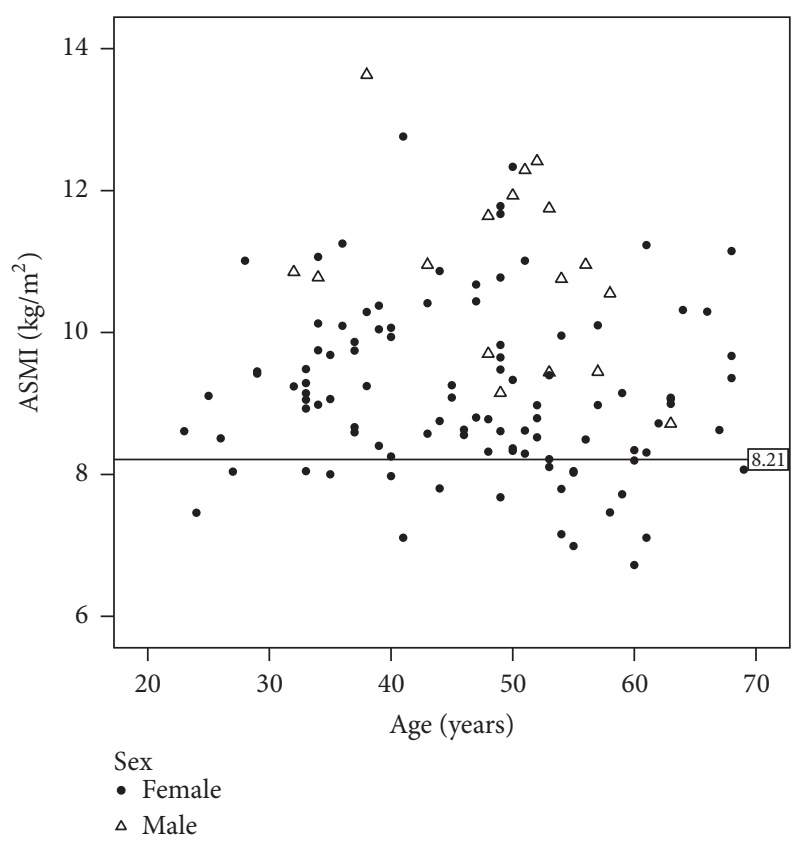

FIGURE 2: Variability of appendicular skeletal mass index (ASMI) by age (23-69 years) in adults with class II/III obesity $(n=120$, females $=103$ ). The line indicated the 20th percentile of ASMI for females; subjects below this level ranged in age from 24 to 69 years.

Our findings support the use of a combined definition of body mass or FM to a measure of sarcopenia for the identification of sarcopenic obesity in this cohort of younger adults with class II/III obesity. Considering measures of ASM with weight [31, 32], BMI [8], FMI [18], or FM [29, 33], our prevalence of sarcopenia ranged from 12.6 to $84.5 \%$ for females and 17.6 to $100 \%$ for males. Likewise, in the Newman et al. [29] study, higher prevalence rates were observed for both sexes using a method in which ASM was considered in relation to height and FM compared to none using nonadjusted ASMI cut points. The authors concluded this technique captured the effect of both LST (as ASM) and high FM simultaneously, therefore identifying a greater proportion of people with obesity as being sarcopenic. Our findings are consistent with their results and highlight the potential importance of considering FM with LST indices together when evaluating sarcopenia in people with obesity.

We were able to identify three body composition phenotypes using the Prado et al. [18] previously established cut points, where age, sex, and BMI-specific reference curves were created to define body composition phenotypes (FMI and ASMI above or below the 50th percentile). As the 50th percentile was used, the terms "obesity" and "sarcopenia" were avoided with individuals being classified using a combination of high/low adiposity and high/low muscularity. The concurrent high adiposity and low muscularity are the "sarcopenic obesity-like" phenotype with an observed population prevalence of $10.3 \%$ in females and $15.2 \%$ in males. Although subjects $>136 \mathrm{~kg}$ were excluded from that study thereby limiting the reference data, applying this method to the current study cohort produced similar results, identifying

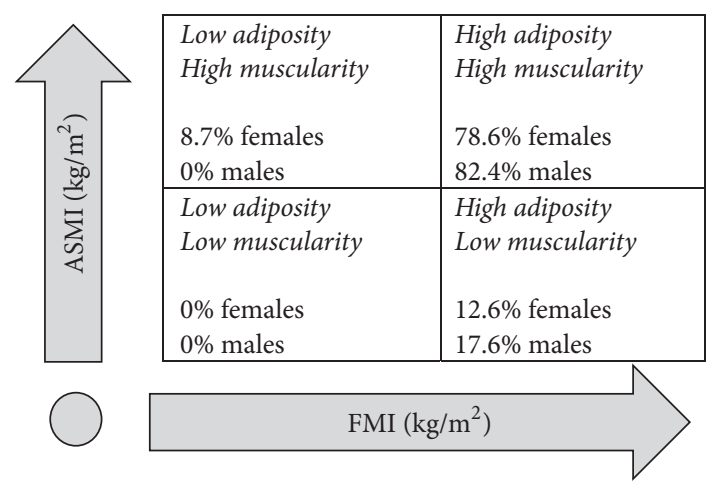

FIGURE 3: Body composition phenotype, by decile groups of appendicular skeletal mass index (ASMI) and fat mass index (FMI), for adults with class II/III obesity $(n=120$, females $=103)$ [18]

$12.6 \%$ of females and $17.6 \%$ of males with sarcopenic obesity. Similar to Siervo et al. [33] FM: FFM ratio reference curves in our study found females had a higher FM:FFM ratio than males. Notably, the current study included subjects with higher weights, with $17.5 \%$ of females and $41.2 \%$ of males $>136 \mathrm{~kg}$. The load-capacity model is a novel method to identify low LST relative together with excess FM in subjects with class II/III obesity.

Although our sample size of males was small, their prevalence of sarcopenia was higher than females for all definitions except the Newman et al. residual method [29], where the prevalence was similar. The prevalence of sarcopenia by sex is controversial with some studies reporting higher prevalence among males, others among females and some finding no differences [35].

An important consideration for any definition is to understand the characteristics of the group from which the cut points were derived. Notably, some definitions were developed from European or Asian cohorts that are ethnically different from a North American population. Widely recognized differences in body composition among different ethnicities preclude a direct comparison of sarcopenic obesity prevalence among different studies.

In the absence of a young reference group, cohort-specific cut points were used using the lowest one [30] or two [28, 29] quintiles for ASMI. Applying this approach to our dataset, our cohort-specific cut points were much higher than previously published ones, again highlighting that cut points derived from other cohorts or nonspecific populations (i.e., older adults, individuals without obesity) may either fail to detect or underestimate the prevalence of sarcopenic obesity in adults with class II/III obesity. Contrary to expectations, the prevalence of sarcopenia was not higher among individuals $\geq 65$ years compared to those $<65$ years [18]. Indeed, we reported ASMI was highly variable across the age spectrum; only one of the 23 individuals with an ASMI below the 20th percentile for this cohort was older than 65 years (Figure 2).

The large variability of LST (Figures 1(a) and 1(b)) in individuals with the same body size represents a clinical challenge for determining nutritional requirements. For example, protein and energy needs are often determined based 
on body weight, yet, considering lean mass drives protein requirements, people with the same body weight can receive varying amounts of protein per unit of lean mass (LST), a concept previously explored $[40,41]$. In the selected example on Figure 1(b), if protein requirements were assessed as $1 \mathrm{~g} / \mathrm{kg}$ actual body weight $(116 \mathrm{~kg})$, the estimated amount of dietary protein would be equivalent to 1.6 to $2.2 \mathrm{~g} / \mathrm{kg} \mathrm{LST}$.

Data on body composition of adults with class II/III obesity is limited, especially of those with BMI $>40 \mathrm{~kg} / \mathrm{m}^{2}$. One barrier is related to equipment limitations [42]. Individuals with class III obesity not only have increased weights, but increased body dimensions such as height or supine width. Although there are large body composition data sets available, subjects above $136 \mathrm{~kg}$ were excluded due to equipment limitations [36]. Recent DXA equipment improvements, such as the Lunar iDXA (GE Healthcare) and Discovery/Horizon models (Hologic, Inc.), have increased scan area widths and weight capacities, improving the capability to assess more people with obesity.

Notably, this study was completed prior to initiation of obesity treatment at the clinic. Weight loss is associated with reductions in both FM and LST, with weight regain predominately as FM [10]. If people with low LST are not identified as such, initiating obesity treatments targeted to reduce weight can further reduce LST, thereby either creating or worsening a sarcopenic state.

Limitations of our study include our ambulatory cohort seeking obesity treatment, which may not reflect all adults with obesity or other care settings (i.e., acute care, long term care). Although the representation of males in the current study appears low, it is comparable to other studies conducted in this clinic $[43,44]$. In general, males tend to be underrepresented in obesity treatment studies $[45,46]$. Additionally, we were unable to explore definitions of sarcopenia using a measure of muscle function, as these were not collected as part of patient's initial assessment.

\section{Conclusions}

Sarcopenia was present in our cohort but masked by obesity. Basic anthropometric measurements alone are inadequate to identify sarcopenia and hence sarcopenic obesity in these individuals. Sophisticated tools such as DXA may be needed to identify and profile LST of adults with class II/II obesity and could be implemented as part of clinical assessment. The inclusion of measures of FM and body size in the definition of sarcopenic obesity identifies a greater proportion of individuals with this abnormal body composition phenotype compared to stand-alone definition of low lean mass. Different diagnostic criteria should be tested in prospective studies investigating the risk prediction for metabolic, functional, and clinical parameters of these adults with class II/III obesity.

\section{Disclosure}

A version of this manuscript was based upon Carlene Johnson Stoklossa's Master's thesis [47].

\section{Conflicts of Interest}

The authors declare that there are no conflicts of interest regarding the publication of this paper.

\section{Acknowledgments}

The authors wish to acknowledge Vickie Baracos, Tasuku Terrada, and Sunita Ghosh and the physicians, staff, and patients of the Edmonton Adult Bariatric Specialty Clinic for their support and generosity of their time and expertise.

\section{References}

[1] M. De Luca, L. Angrisani, J. Himpens et al., "Indications for surgery for obesity and weight-related diseases: position statements from the International Federation for the Surgery of Obesity and Metabolic Disorders (IFSO)," Obesity Surgery, vol. 26, no. 8, pp. 1659-1696, 2016.

[2] World Health Organization Consultation on Obesity, Obesity: Preventing and Managing the Global Epidemic: A Report of the WHO Consultation on Obesity, World Health Organization, Geneva, Switzerland, 1998.

[3] K. M. Flegal, D. Kruszon-Moran, M. D. Carroll, C. D. Fryar, and C. L. Ogden, "Trends in obesity among adults in the United States, 2005 to 2014," Journal of the American Medical Association, vol. 315, no. 21, pp. 2284-2291, 2016.

[4] Statistics Canada, "Table 105-0501- Health indicator profile, annual estimates, by age group and sex, Canada, provinces, territories, health regions (2013 boundaries) and peer groups, occasional, CANSIM (database)," 2013 http://www5.statcan.gc .ca/cansim/a26?lang=eng\&id=1050501.

[5] T. Navaneelan and T. Janz, "Adjusting the scales: obesity in the Canadian population after correcting for respondent bias," 2014.

[6] C. M. M. Prado and S. B. Heymsfield, "Lean tissue imaging: a new era for nutritional assessment and intervention," Journal of Parenteral and Enteral Nutrition, vol. 38, no. 8, pp. 940-953, 2014.

[7] R. N. Baumgartner, K. M. Koehler, D. Gallagher et al., "Epidemiology of sarcopenia among the elderly in New Mexico," American Journal of Epidemiology, vol. 147, no. 8, pp. 755-763, 1998.

[8] J. A. Batsis, T. A. Mackenzie, F. Lopez-Jimenez, and S. J. Bartels, "Sarcopenia, sarcopenic obesity, and functional impairments in older adults: National Health and Nutrition Examination Surveys 1999-2004," Nutrition Research, vol. 35, no. 12, pp. 10311039, 2015.

[9] I. H. Rosenberg, "Sarcopenia: origins and clinical relevance," Journal of Nutrition, vol. 127, no. 5, pp. 990S-991S, 1997.

[10] C. M. M. Prado, J. C. K. Wells, S. R. Smith, B. C. M. Stephan, and M. Siervo, "Sarcopenic obesity: a critical appraisal of the current evidence," Clinical Nutrition, vol. 31, no. 5, pp. 583-601, 2012.

[11] S. Stenholm, D. Alley, S. Bandinelli et al., "The effect of obesity combined with low muscle strength on decline in mobility in older persons: results from the InCHIANTI Study," International Journal of Obesity, vol. 33, no. 6, pp. 635-644, 2009.

[12] J. A. Batsis, S. Singh, and F. Lopez-jimenez, "Anthropometric measurements and survival in older Americans: results from the third national health and nutrition examination survey," Journal of Nutrition, Health and Aging, vol. 18, no. 2, pp. 123-130, 2014. 
[13] A. J. Cruz-Jentoft, J. P. Baeyens, J. M. Bauer et al., "Sarcopenia: European consensus on definition and diagnosis," Age and Ageing, vol. 39, no. 4, pp. 412-423, 2010.

[14] I. Janssen, S. B. Heymsfield, and R. Ross, "Low relative skeletal muscle mass (sarcopenia) in older persons is associated with functional impairment and physical disability," Journal of the American Geriatrics Society, vol. 50, no. 5, pp. 889-896, 2002.

[15] R. H. Demling, "Nutrition, anabolism, and the wound healing process: an overview," Eplasty, vol. 9, article e9, 2009.

[16] S. Tyrovolas, A. Koyanagi, B. Olaya et al., "Factors associated with skeletal muscle mass, sarcopenia, and sarcopenic obesity in older adults: A Multi-Continent Study," Journal of Cachexia, Sarcopenia and Muscle, pp. 312-321, 2016.

[17] P. Cherin, E. Voronska, N. Fraoucene, and C. de Jaeger, "Prevalence of sarcopenia among healthy ambulatory subjects: the sarcopenia begins from 45 years," Aging Clinical and Experimental Research, vol. 26, no. 2, pp. 137-146, 2014.

[18] C. M. M. Prado, M. Siervo, E. Mire et al., "A population-based approach to define body-composition phenotypes," American Journal of Clinical Nutrition, vol. 99, no. 6, pp. 1369-1377, 2014.

[19] H. J. Pownall, G. A. Bray, L. E. Wagenknecht et al., "Changes in body composition over 8 years in a randomized trial of a lifestyle intervention: The Look AHEAD Study," Obesity, vol. 23, no. 3, pp. 565-572, 2015.

[20] C. F. Nicoletti, J. S. Camelo, J. E. Dos Santos, J. S. Marchini, W. Salgado, and C. B. Nonino, "Bioelectrical impedance vector analysis in obese women before and after bariatric surgery: changes in body composition," Nutrition, vol. 30, no. 5, pp. 569574, 2014.

[21] J. A. Batsis, L. K. Barre, T. A. Mackenzie, S. I. Pratt, F. LopezJimenez, and S. J. Bartels, "Variation in the prevalence of sarcopenia and sarcopenic obesity in older adults associated with different research definitions: dual-energy X-ray absorptiometry data from the national health and nutrition examination survey 1999-2004," Journal of the American Geriatrics Society, vol. 61, no. 6, pp. 974-980, 2013.

[22] M. P. Rothney, R. J. Brychta, E. V. Schaefer, K. Y. Chen, and M. C. Skarulis, "Body composition measured by dual-energy x-ray absorptiometry half-body scans in obese adults," Obesity, vol. 17, no. 6, pp. 1281-1286, 2009.

[23] P. A. Tataranni and E. Ravussin, "Use of dual-energy Xray absorptiometry in obese individuals," American Journal of Clinical Nutrition, vol. 62, no. 4, pp. 730-734, 1995.

[24] C. D. Fryar, Q. Gu, and C. L. Ogden, "Anthropometric reference data for children and adults: United States, 2007-2010," Vital and Health Statistics. Series 11, Data from the National Health Survey, vol. 11, no. 252, pp. 1-48, 2007.

[25] Government of Alberta, "Demographic Spotlight-The visible minority population: Recent trends in Alberta and Canada Aug 2011," http://www.finance.alberta.ca/aboutalberta/osi/demographics/ Analysis-and-Reports/2011/2011-0831-visible-minority-populationtrends.pdf.

[26] Province of Alberta, "Freedom of Information and Protection of Privacy Act: Revised Statutes of Alberta 2000, Chapter F-25 current as of Dec 11, 2015 ; Freedom of Information and Protection of Privacy Regulation, Alberta Regulation 200/1995 with amendments up to and including Alberta Regulation 49/2015," Edmonton: Queen's Printer; 2015.

[27] E. Zoico, V. Di Francesco, J. M. Guralnik et al., "Physical disability and muscular strength in relation to obesity and different body composition indexes in a sample of healthy elderly women," International Journal of Obesity, vol. 28, no. 2, pp. 234241, 2004.

[28] T. N. Kim, S. J. Yang, H. J. Yoo et al., "Prevalence of sarcopenia and sarcopenic obesity in Korean adults: the Korean sarcopenic obesity study," International Journal of Obesity, vol. 33, no. 8, pp. 885-892, 2009.

[29] A. B. Newman, V. Kupelian, M. Visser et al., "Sarcopenia: alternative definitions and associations with lower extremity function," Journal of the American Geriatrics Society, vol. 51, no. 11, pp. 1602-1609, 2003.

[30] D. R. Bouchard, I. J. Dionne, and M. Brochu, "Sarcopenic/ obesity and physical capacity in older men and women: data from the nutrition as a determinant of successful aging (NuAge)-The Quebec Longitudinal Study," Obesity, vol. 17, no. 11, pp. 2082-2088, 2009.

[31] M. E. Levine and E. M. Crimmins, "The impact of insulin resistance and inflammation on the association between sarcopenic obesity and physical functioning," Obesity, vol. 20, no. 10, pp. 2101-2106, 2012.

[32] C. Oh, S. Jho, J.-K. No, and H.-S. Kim, "Body composition changes were related to nutrient intakes in elderly men but elderly women had a higher prevalence of sarcopenic obesity in a population of Korean adults," Nutrition Research, vol. 35, no. 1, pp. 1-6, 2015.

[33] M. Siervo, C. M. Prado, E. Mire et al., "Body composition indices of a load-capacity model: gender-and BMI-specific reference curves," Public Health Nutrition, vol. 18, no. 7, pp. 1245-1254, 2015.

[34] L. M. Donini, E. Poggiogalle, S. Migliaccio, A. Aversa, and A. Pinto, "Body composition in sarcopenic obesity: systematic review of the literature," Mediterranean Journal of Nutrition and Metabolism, vol. 6, no. 3, pp. 191-198, 2013.

[35] A. J. Cruz-Jentoft, F. Landi, S. M. Schneider et al., "Prevalence of and interventions for Sarcopenia in ageing adults: a systematic review. Report of the international Sarcopenia initiative (EWGSOP and IWGS)," Age and Ageing, vol. 43, no. 6, pp. 48-759, 2014.

[36] T. L. Kelly, K. E. Wilson, and S. B. Heymsfield, "Dual energy $\mathrm{X}$-ray absorptiometry body composition reference values from NHANES," PLoS ONE, vol. 4, no. 9, Article ID e7038, 2009.

[37] R. N. Baumgartner, S. J. Wayne, D. L. Waters, I. Janssen, D. Gallagher, and J. E. Morley, "Sarcopenic obesity predicts instrumental activities of daily living disability in the elderly," Obesity Research, vol. 12, no. 12, pp. 1995-2004, 2004.

[38] J. A. Batsis, T. A. Mackenzie, L. K. Barre, F. Lopez-Jimenez, and S. J. Bartels, "Sarcopenia, sarcopenic obesity and mortality in older adults: results from the National Health and Nutrition Examination Survey III," European Journal of Clinical Nutrition, vol. 68, no. 9, pp. 1001-1007, 2014.

[39] C. Beaudart, J.-Y. Reginster, J. Slomian, F. Buckinx, M. Locquet, and O. Bruyère, "Prevalence of sarcopenia: the impact of different diagnostic cut-off limits," Journal of Musculoskeletal Neuronal Interactions, vol. 14, no. 4, pp. 425-431, 2014.

[40] C. M. Prado, S. J. Cushen, C. E. Orsso, and A. M. Ryan, "Sarcopenia and cachexia in the era of obesity: clinical and nutritional impact," Proceedings of the Nutrition Society, vol. 75, no. 2, pp. 188-198, 2016.

[41] C. Geisler, C. Prado, and M. Müller, "Inadequacy of body weight-based recommendations for individual protein intakelessons from body composition analysis," Nutrients, vol. 9, no. 1, article no. 23, 2017. 
[42] C. A. Johnson Stoklossa, M. Forhan, R. S. Padwal, M. C. Gonzalez, and C. M. Prado, "Practical considerations for body composition assessment of adults with class II/III obesity using bioelectrical impedance analysis or dual-energy X-ray absorptiometry," Current Obesity Reports, vol. 5, no. 4, pp. 389396, 2016.

[43] C. B. Agborsangaya, S. R. Majumdar, A. M. Sharma, E. W. Gregg, and R. S. Padwal, "Multimorbidity in a prospective cohort: prevalence and associations with weight loss and health status in severely obese patients," Obesity, vol. 23, no. 3, pp. 707712, 2015.

[44] R. S. Padwal, C. F. Rueda-Clausen, A. M. Sharma et al., "Weight loss outcomes in wait-listed, medically managed, and surgically treated patients enrolled in a population-based bariatric program prospective cohort study," Medical Care, vol. 52, no. 3, pp. 208-215, 2014.

[45] H. F. Fuchs, R. C. Broderick, C. R. Harnsberger et al., "Benefits of bariatric surgery do not reach obese men," Journal of Laparoendoscopic and Advanced Surgical Techniques, vol. 25, no. 3, pp. 196-201, 2015.

[46] S. L. Pagoto, K. L. Schneider, J. L. Oleski, J. M. Luciani, J. S. Bodenlos, and M. C. Whited, "Male inclusion in randomized controlled trials of lifestyle weight loss interventions," Obesity, vol. 20, no. 6, pp. 1234-1239, 2012.

[47] C. A. Johnson Stoklossa, Prevalence and characteristics of sarcopenic obesity in adults with class II/III obesity [M.S. thesis], University of Alberta, Alberta, Canada, 2016. 


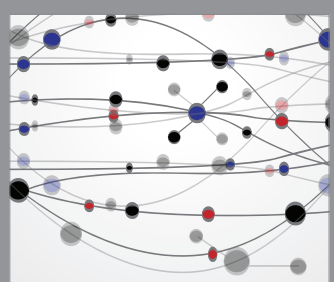

The Scientific World Journal
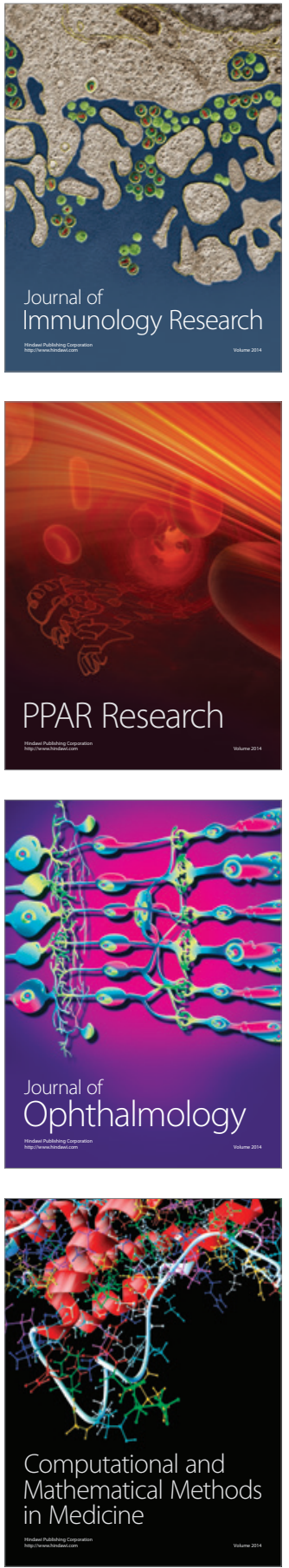

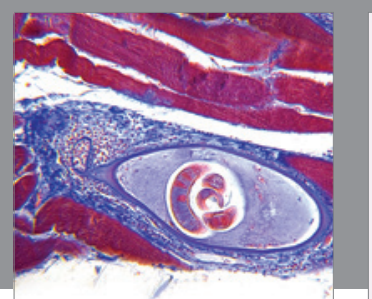

Gastroenterology Research and Practice
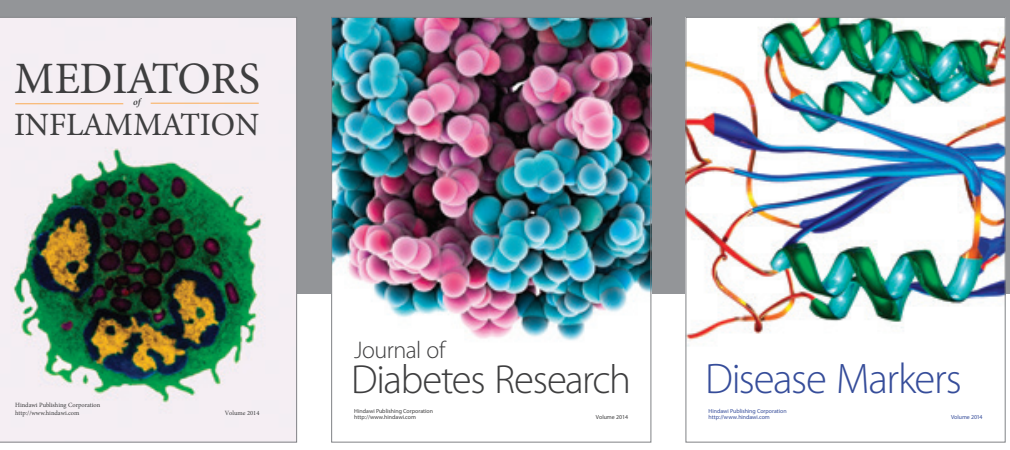

Disease Markers

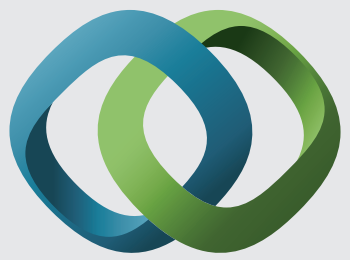

\section{Hindawi}

Submit your manuscripts at

https://www.hindawi.com
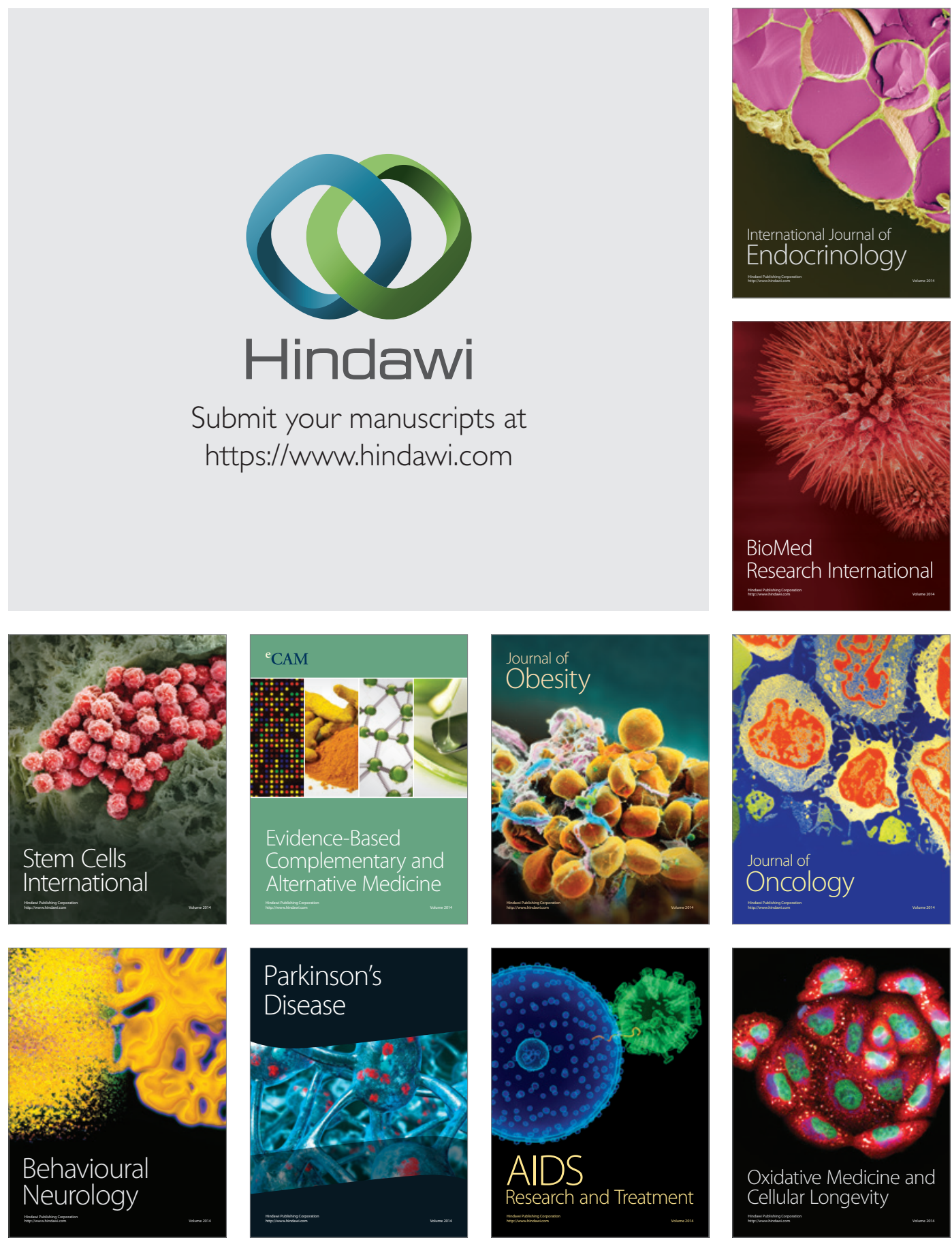